\title{
Perceptions des professionnels de la santé face à la présence de la famille lors des manœuvres de réanimation : une revue intégrative des écrits
}

\author{
Quentin Bevillard-Charrière'1, inf. M. Sc., Steve Gagné2, inf., MScN, CNCC (C), Laurence Bernard³, inf. Ph.D \\ ${ }^{1}$ Faculté des Sciences Infirmières, Université de Montréal, quentin.bevillard-charriere@umontreal.ca \\ ${ }^{2}$ Conseiller en soins spécialisés - Développement de la pratique et des compétences, Chercheur-Professionnel de la santé au Centre de recherche du \\ CHUM, Centre Hospitalier de I'Université de Montréal, Infirmier clinicien spécialisé associé, Faculté des sciences infirmières, Université de Montréal, \\ steve.gagne.chum@ssss.gouv.qc.ca \\ ${ }^{3}$ Professeure agrégée, Faculté des Sciences Infirmières, Université de Montréal, laurence.bernard@umontreal.ca
}

\section{Résumé}

Contexte : l'inclusion des familles lors d'une réanimation cardiorespiratoire constitue un défi clinique. En effet, les familles sont souvent absentes des protocoles de réanimation qui ciblent les interventions de l'équipe interprofessionnelle composée de médecins, d'infirmiers, d'inhalothérapeutes et de préposés aux bénéficiaires. La littérature scientifique existante parait peu étoffée sur la perception de l'équipe interprofessionnelle face à l'inclusion des familles durant la réanimation.

Objectif : le but de cette revue intégrative est de recenser des écrits traitant de la perception des membres de l'équipe interprofessionnelle face à la présence de la famille lors des manœuvres de réanimation.

Méthodologie : cette revue intégrative des écrits a été réalisée en consultant les bases de données Cumulative Index to Nursing Information and Allied Health Literature (CINAHL), PsycINFO, Medline, Web of Science, Cochrane et Joanna Briggs Institute (JBI). Les données ont été organisées selon les trois thèmes qu'identifient Twibell et al. (2008) dans leurs travaux : les bénéfices perçus par les professionnels de la santé, les risques perçus et le niveau de confiance en la pratique professionnelle.

Résultats : sur un total de 1910 écrits répertoriés, 23 articles scientifiques ont été retenus. Les trois thèmes identifiés dans les travaux de Twibell et al. (2008) ont servi de base à l'analyse des écrits. Cette dernière a été effectué à l'aide d'une analyse thématique. La littérature recensée aborde la perception principalement des infirmiers et des médecins, mais très peu des autres membres de l'équipe interprofessionnelle. Malgré les risques ou les appréhensions ressentis par les infirmiers et les médecins, la présence de la famille durant les manœuvres demeure bénéfique pour celle-ci.

Limites : cette revue intégrative n'a pas retenu d'article ciblant le point de vue des gestionnaires ou de décideurs politiques ni le point de vue de l'équipe interprofessionnelle œuvrant dans les urgences pédiatriques. De plus, la méthode d'analyse des données basée sur les thèmes de Twibell et al. (2008) entraîne un biais dans l'émergence d'autres thèmes. Enfin, les perceptions de l'équipe interprofessionnelle n'ont pas été rattachées au milieu d'exercice. 
Conclusion : les résultats de cette revue intégrative offrent des pistes d'interventions qui permettront d'améliorer la pratique d'inclusion des familles lors des manœuvres de réanimation. Une attention particulière devrait être portée sur la formation initiale et continue des professionnels de la santé. De plus, cet article permet un début de réflexion chez les gestionnaires et décideurs afin de promouvoir une culture collaborative ainsi qu'une approche centrée sur le patient.

Mots-clés : équipe interprofessionnelle, réanimation, urgence, famille, revue intégrative

\section{Introduction}

A département d'urgence, toutes manœuvres de réan$A$ imation cardiorespiratoire impliquent une charge émotionnelle pour la personne, sa famille et l'équipe interprofessionnelle. Toutefois, de nombreuses études révèlent le souhait de la famille à vouloir être présente auprès de leurs proches lors d'une pareille situation critique de soins (Meyers et al., 2000; Mian et al., 2007). Plusieurs chercheurs rapportent de nombreux avantages de cette inclusion de la famille tels que cette dernière représente, une source de renseignement pour l'équipe interprofessionnelle (Jensen et Kosowan, 2011; Knott et Kee, 2005). D’autres auteurs soulignent que la présence familiale permettrait d'humaniser cette situation de soins critiques (Chapman et al., 2014; McClement et al., 2009; Porter et al., 2014). C'est pourquoi, l'ENA (Emergency Nurses Association) a émis en 2007, des recommandations dans l'objectif de développer et soutenir cette pratique d'inclusion aux urgences (Emergency Nurses Association [ENA], 2012). Néanmoins malgré ces recommandations, de nombreuses études révèlent qu'il ne s'agit toujours pas d'une pratique courante. Les opinions des professionnels de la santé sont partagées et une majorité indique que l'inclusion des familles va à l'encontre de la pratique habituelle d'exclusion des familles (Fisher et al., 2008; MacLean et al., 2003; Mason, 2003). Face à cette réticence des professionnels de la santé, il devient important de s'intéresser aux perceptions de l'équipe interprofessionnelle face à la présence de la famille lors des manœuvres de réanimation. La présence de la famille en réanimation équivaut à la présence de la famille au sein de l'environnement où sont prodigués les manœuvres de réanimation (Twibell et al., 2018). Cette présence est synonyme de contact visuel ou physique avec le patient lorsque les manœuvres de réanimation sont en cours (Twibell et al., 2018). Ainsi, le but de l'article est de dresser un portrait des perceptions de l'équipe interprofessionnelle face à la présence de la famille lors des manœuvres de réanimation au département d'urgence.

\section{Méthode}

Cette revue intégrative des écrits a été réalisée selon la méthode de Whittemore et Knafl (2005) comprenant cinq étapes : l'identification du problème, la recherche documentaire, l'évaluation des articles, l'analyse des écrits, la présentation d'une synthèse des écrits.
La stratégie de recherche a mobilisé trois principaux concepts : interprofessional, family et resuscitation. Un repérage d'articles scientifiques a été effectué via les bases de données Cumulative Index to Nursing Information and Allied Health Literature (CINAHL), PsycINFO, Medline, Web of Science, Cochrane et Joanna Briggs Institute (JBI). Pour être inclus, les écrits devaient : être publiés en langues anglaises ou françaises ; études qualitatives/quantitatives/mixte/revue de la littérature ; publiés après 2005 afin de cibler des écrits récents ; traiter de la présence familiale lors des manœuvres de réanimation ou aborder les perceptions des professionnels de la santé en lien avec la présence de la famille lors des manœuvres de réanimation. Les écrits reflétant la réalité des urgences pédiatriques et les études provenant d'un pays dont la pratique clinique ne s'apparente pas à la réalité nord-américaine ont été exclus. L'évaluation des articles a été effectuée à l'aide d'une grille analysant la pertinence, la rigueur et la méthodologie de l'article. Cette grille s'appuie sur le livre de Loiselle et Profetto-McGrath (2007). La littérature grise n'a pas été incluse sur recommandation d'un bibliothécaire. En effet, à titre d'exemple, les protocoles et politiques d'inclusion ou d'exclusion des familles sont habituellement des documents non publiés, internes à l'organisation de soin.

\section{Figure 1. Processus de sélection des écrits}

1910 écrits potentiels répertoriés dans les basses de données électroniques

190 articles triés

23 écrits inclus dans la revue intégrative

\section{0 articles exclus}

- Publiés en langue autre que l'anglais ou le français

- Publié avant 2005

- Documents traitants de la clientèle pédiatrique

- Pays non pertinent

- Ne répondant pas aux critères d'inclusion soit : document traitant de la présence familiale en réanimation

162 articles exclus

Ne correspondant pas au but de la revue intégrative

\section{Analyses des données}

L'analyse des données a été construite autour des trois thèmes identifiés dans les travaux de Twibell et al. (2008), grâce à une analyse thématique des données (Paillé et Muchielli, 2016). Ce type d'analyse a été efficace pour identifier les perceptions de l'équipe interprofessionnelle face à la présence de la famille lors des manœuvres de réanimation, en les mettant en lien avec les trois thèmes identifiés par Twibbel et al. (2008). Ainsi, les résultats de la présente revue intégrative mettent en lumière les bénéfices perçus par les infirmiers et médecins principalement face à la présence de la famille ; les risques perçus par ces derniers face à la présence de la famille et leur niveau de confiance en la pratique professionnelle face à la présence de celle-ci (voir tableau 1). 
Tableau 1. Bénéfices et risques perçus par les infirmiers/médecins selon différentes sphères

\begin{tabular}{l} 
BÉNÉFICES PERÇUS \\
\hline Patient/Famille \\
\hline - Lien d'attachement entre le patient et ses \\
proches maintenu (Fell, 2009; Holzhauser \\
et Finucane, 2008; Howlett et al., 2010; \\
McClement et al., 2009) \\
- Prise de conscience des familles de l'ampleur \\
des soins fournis (Chapman et al., 2014; Fell, \\
2009; Holzhauser et Finucane, 2008; Howlett \\
et al., 2010; Knott et Kee, 2005; McClement et \\
al., 2009; Porter et al., 2014; Tudor et al., 2014) \\
- Diminution de l’anxiété de la famille devant \\
une compréhension de la gravité de la situa- \\
tion (Jensen et Kosowan, 2011) \\
- Aide à l'acceptation de la fin de la réanimation \\
(Gomes et al., 2019) \\
- Aide au processus du deuil (Axelsson et al., \\
2010; Chapman et al., 2014; García-Martínez \\
et Meseguer-Liza, 2018; Howlett et al., 2010; \\
Knott et Kee, 2005) \\
- Diminution d'un stress post-traumatique \\
(Jabre et al., 2013)
\end{tabular}

RISQUES PERÇUS

\begin{tabular}{|c|c|c|}
\hline Patient/Famille & Infirmiers/médecins & Environnement \\
\hline $\begin{array}{l}\text { - Présence synonyme de fardeau émotionnel } \\
\text { (García-Martínez et Meseguer-Liza, 2018; } \\
\text { Holzhauser et Finucane, 2007, 2008; Howlett } \\
\text { et al., 2010; Knott et Kee, 2005; Miller et } \\
\text { Stiles, 2009) } \\
\text { - Traumatisme psychologique (Asencio- } \\
\text { Gutiérrez et Reguera-Burgos, 2017; Fell, 2009; } \\
\text { Howlett et al., 2010; McClement et al., 2009; } \\
\text { Porter et al., 2014; Tudor et al., 2014) } \\
\text { - Mauvaise interprétation de certains actes } \\
\text { médicaux (Knott et Kee, 2005; Tudor et al., } \\
\text { 2014) } \\
\text { - Risque d'une mauvaise adaptation du proces- } \\
\text { sus de deuil (Holzhauser et Finucane, 2007) } \\
\text { Violation de la confidentialité et de la vie } \\
\text { privée du patient (Holzhauser et Finucane, } \\
\text { 2007; Tomlinson et al., 2010) }\end{array}$ & $\begin{array}{l}\text { - Émotions pouvant interférer la réanimation } \\
\text { (Chapman et al., 2014; Holzhauser et Finucane, } \\
\text { 2007; Howlett et al., 2010; Köberich et al., } \\
\text { 2010; Porter et al., 2014; Tomlinson et al., 2010; } \\
\text { Waldemar et Thylen, 2019) } \\
\text { - Manœuvres de réanimation davantage } \\
\text { stressantes (Jensen et Kosowan, 2011) } \\
\text { - Augmentation des poursuites judiciaires } \\
\text { (Axelsson et al., 2010; Fell, 2009; Howlett et al., } \\
\text { 2010; Köberich et al., 2010; McClement et al., } \\
\text { 2009; Porter et al., 2014; Powers et Reeve, 2018; } \\
\text { Tomlinson et al., 2010) } \\
\text { - Difficulté à mettre fin aux manœuvres de réan- } \\
\text { imation (Axelsson et al., 2010; Holzhauser et } \\
\text { Finucane, 2008; Howlett et al., 2010) }\end{array}$ & $\begin{array}{l}\text { - Réduction considérable } \\
\text { de la taille de l'envi- } \\
\text { ronnement de la salle de } \\
\text { réanimation (Chapman } \\
\text { et al., 2014; Holzhauser } \\
\text { et Finucane, 2008; } \\
\text { Howlett et al., 2010; } \\
\text { Knott et Kee, 2005; } \\
\text { McClement et al., 2009; } \\
\text { Waldemar et Thylen, } \\
\text { 2019) }\end{array}$ \\
\hline \multicolumn{3}{|c|}{ NIVEAU DE LA CONFIANCE EN LA PRATIQUE PROFESSIONNELLE } \\
\hline \multicolumn{3}{|c|}{$\begin{array}{l}\text { - Plus le taux de confiance sera élevé, plus les médecins et infirmiers seront en mesure d'inviter la famille (Twibell et al., 2008) } \\
\text { - L'absence d'autorité discrétionnaire est un frein au niveau de confiance (Fulbrook et al., 2005) } \\
\text { - Lorsque l'opinion du professionnel est favorable alors son degré d'aisance augmente (Holzhauser et Finucane, 2007) }\end{array}$} \\
\hline
\end{tabular}




\section{Résultats}

Des 1910 articles potentiels répertoriés, 1720 articles ont été exclus parce qu'ils ne répondaient pas aux critères d'inclusion (Figure 1). Des 190 articles retenus initialement, 158 ont été exclus ne correspondant pas au but de la revue intégrative qui était de recenser des écrits traitants de la perception des professionnels face à la présence de la famille en contexte de réanimation. Finalement, 23 écrits ont été analysés à l'aide d'une grille structurée produite par les auteurs. Cette dernière a été utilisée afin de synthétiser les données issues de l'analyse thématique. Elle regroupe les informations suivantes : la référence de l'article, le pays, le devis, le but, les données pouvant être associées aux bénéfices perçus, aux risques perçus et au niveau de confiance en la pratique professionnelle. Des 23 études retenues, on retrouve une revue intégrative des écrits $(\eta=1)$, des revues de littérature $(\eta=4)$, des études à devis quantitatif $(\eta=11)$, à devis qualitatif $(\eta=5)$, à devis mixte $(\eta=1)$ et un essai contrôlé randomisé $(\eta=1)$. La majorité des études $(\eta=20)$ ont été publiées à partir de 2008 . Cette date correspond à la publication des travaux de Twibell et al. (2008) sur l'élaboration d'un outil validé par ses propriétés psychométriques permettant la mesure des perceptions des infirmiers face à la présence familiale lors d'une réanimation qui a ouvert une voie quant à la recherche infirmière sur l'inclusion de la famille lors des manœuvres de réanimation.

La plupart des études proviennent d'Amérique du Nord soit aux États-Unis $(\eta=10)$ et au Canada $(\eta=2)$. Les autres ont été réalisées en Europe $(\eta=5)$, en Australie $(\eta=5)$ et une en Amérique latine. Le nombre de participants dans les études qualitatives variaient entre 10 et 242 . Pour les études quantitatives les échantillons variaient entre 40 et 570. La majorité des études présentaient un échantillon supérieur à 120. Le nombre des échantillons des études varie grandement pouvant ainsi influencer la généralisation des résultats. Néanmoins, la généralisation n'est pas une fin en soi dans la méthode de revue intégrative des écrits. Les participants étaient soit uniquement des infirmiers $(\eta=12)$, soit des infirmiers et des médecins $(\eta=5)$ et une étude comportait uniquement des médecins et ce, malgré l'inclusion de la notion d'équipe interprofessionnelle dans les critères de sélection des articles et des mots-clés. Les études proviennent des départements d'urgence $(\eta=6)$, des unités de soins intensifs $(\eta=5)$, de toutes les unités confondues de centres hospitaliers $(\eta=4)$, des unités de cardiologie $(\eta=3)$ et une étude a visé les infirmiers membres de l'ENA et de l'AACN (American Association of Critical-Care Nurses).

\section{Thème 1 : Les bénéfices perçus par les infirmiers et médecins face à la présence de la famille.}

Nous constatons, dans la littérature scientifique, de nombreux bénéfices perçus par les infirmiers et les médecins. Selon Twibell et al. (2018), le bénéfice perçu est l'évaluation par un individu de la valeur de l'adoption d'un comportement. Ces bénéfices concernent principalement deux sphères : patient/famille et infirmiers/médecins.

\section{Bénéfices reliés au patient/famille.}

Dix-huit articles évaluent les bénéfices reliés au patient/famille. Les bénéfices perçus par l'équipe pour les patients/familles sont nombreux : ils concernent le réconfort, le soutien affectif, la prise de conscience de l'ampleur des soins, le processus de deuil, l'interruption de la réanimation et l'accompagnement et à la diminution d'apparition de stress post-traumatique.

Dans un premier temps, les bénéfices reliés au patient sont moins présents dans la littérature. Waldemar et Thylen (2019) constatent que les infirmiers $(\eta=189)$ estiment que la présence familiale serait bénéfique pour le patient. Certains auteurs appuient que la présence familiale apporte un réconfort, un soutien affectif chez le patient (Fell, 2009; Holzhauser et Finucane, 2008; Howlett et al., 2010; McClement et al., 2009). D’après ces derniers, la présence familiale permettrait de maintenir le lien d'attachement entre le patient et ses proches.

Dans un second temps, les bénéfices reliés à la famille sont bien documentés. Selon plusieurs auteurs, les infirmiers pensent que la présence familiale lors d'une réanimation permettrait à la famille de réaliser une prise de conscience de l'ampleur des soins fournis pour leur proche ainsi que les efforts déployés par l'équipe soignante (Chapman et al., 2014; Fell, 2009; Holzhauser et Finucane, 2008; Howlett et al., 2010; Knott et Kee, 2005; McClement et al., 2009; Porter et al., 2014; Tudor et al., 2014). Cette prise de conscience permettrait à la famille d'être rassurée sur le fait que tout a été fait pour venir en aide à leur proche (Tudor et al., 2014) et de donner un sens à la situation (Porter et al., 2014). De nombreux auteurs rapportent que la présence familiale permettrait à la famille de savoir que tout a été entrepris pour leur proche (Fulbrook et al., 2005; Gomes et al., 2019; Jensen et Kosowan, 2011; Köberich et al., 2010). De ce fait, l'anxiété de la famille pourrait diminuer et une compréhension de la gravité de la situation pourrait se manifester (Jensen et Kosowan, 2011). Waldemar et Thylen (2019) poursuivent en rapportant que cette présence empêcherait la famille d'avoir une image déformée ou erronée de la situation. Certains auteurs montrent qu'en étant présente auprès de leur proche, la famille serait davantage susceptible d'accepter l'arrêt des manœuvres de réanimation (Howlett et al., 2010; McClement et al., 2009) et que les infirmiers ressentent ce même bénéfice (Fulbrook et al. (2005) ; Köberich et al. (2010). De plus, Gomes et al. (2019) montre que $82 \%$ des infirmiers $(\eta=40)$ estiment que les membres de la famille accepteraient plus facilement la fin du traitement en étant présente. Plusieurs auteurs rapportent la possibilité donnée à la famille d'être présente dans les derniers instants de vie de leur proche leur permettant ainsi de leur dire au revoir durant le processus d'arrêt des manœuvres de réanimation (Axelsson et al., 2010; Fulbrook et al. (2005); Holzhauser et Finucane, 2007; Howlett et al., 2010; Köberich et al. (2010); McClement et al., 2009). Selon de nombreux auteurs, la présence familiale aiderait la famille à entamer le processus de deuil (Axelsson et al., 2010; Chapman et al., 2014; Fulbrook et al., 2005; GarcíaMartínez et Meseguer-Liza, 2018; Howlett et al., 2010; Knott et Kee, 2005; Waldemar et Thylen, 2019).

Suite à une étude, Jabre et al. (2013) rapportent que la fréquence d'apparition d'un stress post-traumatique était significativement plus élevée chez les familles n'ayant pas été présentes lors de la réanimation de leur proche comparativement aux familles ayant assisté à la réanimation. Certains symptômes du stress 
post-traumatique tels que l'anxiété et la dépression étaient davantage présents chez les membres de la famille qui n'ont pas été témoins de la réanimation (Jabre et al., 2013).

\section{Bénéfices reliés aux infirmiers et médecins.}

Quatorze articles ont été inclus dans le sous-thème des bénéfices reliés aux infirmiers et médecins. Les bénéfices reliés aux infirmiers et/ou médecins rapportés dans les écrits sont les suivants : liens famille/soignant renforcés, connaissance rapide des antécédents du patient, et une situation de réanimation humanisée. De nombreux auteurs soulèvent que la présence familiale contribuerait à renforcer les liens entre la famille et l'équipe de soins (Asencio-Gutiérrez et Reguera-Burgos, 2017; Fulbrook et al., 2005; Miller et Stiles, 2009; Porter et al., 2014; Powers et Reeve, 2018). Miller et Stiles (2009) soulèvent que les infirmiers déclarent se sentir appréciées par la famille si cette dernière a pu être présente lors de la réanimation. Ces mêmes auteurs soulignent également une reconnaissance de la famille pour les efforts entrepris par les professionnels renforçant ainsi le lien soignant-famille. Certains auteurs soulignent que la présence familiale représenterait une aide pour les soignants en étant source de renseignement du patient (Chapman et al., 2014; Fell, 2009; Holzhauser et Finucane, 2008; Howlett et al., 2010). Des auteurs (Axelsson et al., 2010; Fulbrook et al., 2005) rapportent que grâce à la présence familiale le rendement de l'équipe serait affecté positivement. D'autres auteurs soutiennent que la présence familiale permettrait d'humaniser le patient et non de le percevoir comme un simple corps à réanimer (Chapman et al., 2014; McClement et al., 2009; Porter et al., 2014). Holzhauser et Finucane (2008) soulignent que la présence de la famille peut être également une source de réconfort pour les infirmiers et les médecins.

\section{Thème 2 : Les risques perçus par les infirmiers et médecins face à la présence de la famille}

Les risques perçus par les infirmiers et les médecins face à la présence de la famille lors d'une réanimation sont bien documentés dans la littérature scientifique. Twibell et al., (2018) définissent le risque perçu comme étant la susceptibilité ressentie par une personne à une menace. L'expérience d'inclusion des familles peut être négative dès lors que le professionnel perçoit des risques à l'inclure.

\section{Risques reliés au patient/famille.}

Quinze articles évaluent les risques reliés à la famille. Les risques perçus par les infirmiers et les médecins reliés au patient/famille sont divers et variés : fardeau émotionnel, interprétation des soins faussée, comportement néfaste, perturbation du processus de deuil, et bris de confidentialité.

De nombreux auteurs soulèvent un fardeau émotionnel pour la famille dû à leur présence pendant la réanimation de leur proche (Fulbrook et al., 2005; García-Martínez et Meseguer-Liza, 2018; Holzhauser et Finucane, 2007, 2008; Howlett et al., 2010; Knott et Kee, 2005; Miller et Stiles, 2009). Köberich et al. (2010) montrent que $24 \%$ des infirmiers $(\eta=394)$ craignent que les membres de la famille puissent souffrir d'effets émotionnels à long terme. Récemment, Waldemar et Thylen (2019) rapportent que 13,2\% des infirmiers et des médecins $(\eta=189)$ ont estimé que la famille souffrira également d'effets émotionnels négatifs. Certains auteurs soulèvent un traumatisme psychologique envisageable sur la famille (Asencio-Gutiérrez et Reguera-Burgos, 2017; Fell, 2009; Howlett et al., 2010; McClement et al., 2009; Porter et al., 2014; Tudor et al., 2014). Jensen et Kosowan (2011) rapportent que $21,9 \%$ des infirmiers et médecins $(\eta=137)$ pensent que l'expérience serait trop traumatisante et dérangeante pour la famille. Des auteurs soulèvent que la présence familiale pourrait engendrer une mauvaise interprétation des proches de certains actes médicaux, pensants même que l'équipe soignante agit avec plus de mal que de bien (Knott et Kee, 2005; Tudor et al., 2014). Un pourcentage de $75 \%$ des infirmiers $(\eta=124)$ de l'étude de Fulbrook et al. (2005) pensent qu'elles pourraient dire des choses qui contrarieraient involontairement les membres de la famille. D'autres auteurs rapportent un comportement possible de la famille caractérisé des proches hors de contrôle, hystériques, pris de panique (Holzhauser et Finucane, 2007; Knott et Kee, 2005). Fell (2009) a relevé que les soignants estimaient qu'un membre de la famille pourrait s'évanouir pendant la réanimation de leur proche. Holzhauser et Finucane (2007) soulèvent un risque de développer une mauvaise adaptation $\mathrm{du}$ processus de deuil. Gomes et al. (2019) rapportent que $12 \%$ des infirmiers $(\eta=40)$ interrogés estiment que la présence familiale prolongerait le deuil. Nombreux sont les auteurs qui rapportent que la confidentialité du patient et de son droit à la vie privée peuvent être violés par la présence familiale (Fulbrook et al., 2005; Holzhauser et Finucane, 2007; Köberich et al. 2010; Tomlinson et al., 2010). Fulbrook et al. (2005), ainsi que Waldemar et Thylen (2019), rapportent respectivement que $36,9 \%$ des infirmiers $(\eta=124)$ de la première étude ont la conviction que la présence familiale n'est pas bénéfique pour le patient. Tandis que $31,4 \%$ des infirmiers $(\eta=124)$ et $55,6 \%$ des médecins $(\eta=65)$ de la deuxième étude ont également cette perception. Somme toute, les risques perçus par les infirmiers et les médecins reliés au patient/famille sont nombreux.

\section{Risques reliés aux infirmiers et médecins.}

Dix-huit articles évaluent les risques reliés aux infirmiers et médecins. Plusieurs risques reliés aux infirmiers et médecins sont rapportés dans les écrits : interférence de la réanimation, poursuites judiciaires, l'arrêt de la réanimation difficile. Nombreux auteurs soulèvent que les émotions éprouvées par la famille peuvent interférer la réanimation : l'anxiété (Asencio-Gutiérrez et Reguera-Burgos, 2017; Fell, 2009; Howlett et al., 2010; Knott et Kee, 2005; McClement et al., 2009; Porter et al., 2014; Waldemar et Thylen, 2019), du stress (Chapman et al., 2014; Holzhauser et Finucane, 2007; Howlett et al., 2010; Köberich et al., 2010; Porter et al., 2014; Tomlinson et al., 2010; Waldemar et Thylen, 2019), de la peur (Holzhauser et Finucane, 2007; Howlett et al., 2010). Jensen et Kosowan (2011) soulèvent que $61,5 \%$ des membres des médecins et infirmiers $(\eta=137)$ pensent que la présence familiale rendrait la réanimation plus stressante pour les membres de l'équipe. McClement et al. (2009) mentionnent que les soignants évoquent une perte de confiance des compétences professionnelles devant la famille présente pendant la réanimation.

Plusieurs auteurs soulèvent une peur des soignants que la présence familiale puisse engendrer une augmentation des poursuites judiciaires contre les membres de l'équipe de 
réanimation (Axelsson et al., 2010; Fell, 2009; Howlett et al., 2010; Köberich et al., 2010; McClement et al., 2009; Porter et al., 2014; Powers et Reeve, 2018; Tomlinson et al., 2010). Fulbrook et al. (2005) ont un taux de $26 \%$ d'infirmiers $(\eta=124)$ estimant que des malentendus pourraient entrainer une augmentation des poursuites. Fell (2009) à montrer que les prestataires de soins pensent que les interventions des soignants peuvent être mal interprétées amenant les familles à croire que l'équipe de réanimation est incompétente. Plusieurs auteurs rapportent que les professionnels ont tendance à faire preuve de laxisme et utiliser l'humour afin de diminuer la pression lors des manœuvres de réanimation (Axelsson et al., 2010; McClement et al., 2009; Miller et Stiles, 2009). D'après ces mêmes auteurs, ceci pourrait encourager les membres de la famille à engager des poursuites judiciaires si l'issue de la réanimation est fatale. Des auteurs soulèvent une difficulté possible à mettre fin à la réanimation due à la présence familiale (Axelsson et al., 2010; Fulbrook et al., 2005; Holzhauser et Finucane, 2008; Howlett et al., 2010). Köberich et al. (2010) montrent que 23\% des infirmiers $(\eta=394)$ pensent que la présence familiale provoquerait des tentatives de réanimation inutiles. Nombreuses études soulèvent que la famille pourrait interférer au processus de réanimation en perturbant l'équipe de soins (Axelsson et al., 2010; Chapman et al., 2014; Howlett et al., 2010; Jensen et Kosowan, 2011; Köberich et al., 2010; Porter et al., 2014; Tomlinson et al., 2010). Des auteurs montrent que cette interférence pourrait perturber les soins de réanimation (Jensen et Kosowan, 2011; McClement et al., 2009; Powers et Reeve, 2018) ou augmenter la charge de travail de l'équipe de réanimation (Chapman et al., 2014). Cette interférence pourrait procurer un sentiment d'inconfort (Knott et Kee, 2005) ou d'un sentiment d'inadéquation (McClement et al., 2009) chez les infirmiers. Des auteurs rapportent que l'observation de la famille pourrait entrainer une distraction possible des soignants (Fell, 2009; Powers et Reeve, 2018). D'autres auteurs parlent d'une concentration difficile de l'équipe (Axelsson et al., 2010; Fulbrook et al., 2005). Récemment Waldemar et Thylen (2019) obtiennent un taux de $20,5 \%$ de la part des infirmiers $(\eta=124)$ et $41,2 \%$ chez les médecins $(\eta=65)$ par rapport à cette perception.

\section{Risques reliés à l'environnement de l'aire de réanimation.}

Sept articles évaluent les risques reliés à l'environnement. Globalement, peu d'auteurs soulignent les risques reliés à l'environnement de l'aire de réanimation. Néanmoins, quelques auteurs rapportent une réduction considérable de la taille de l'environnement de la salle de réanimation (Chapman et al., 2014; Holzhauser et Finucane, 2008; Howlett et al., 2010; Knott et Kee, 2005; McClement et al., 2009; Waldemar et Thylen, 2019). Köberich et al. (2010) soulèvent que 42 infirmiers $(\eta=394)$ pensent que la superficie des chambres de réanimation n'est pas assez spacieuse pour permettre la présence familiale qui réduirait davantage l'environnement. Malgré ces considérations environnementales, il est important de souligner que les auteurs, dans la plupart des études, rapportent des taux inférieurs à 50\% concernant les risques perçus par les infirmiers et les médecins. Ainsi, une majorité des soignants ne perçoivent pas de risques dû à la présence familiale lors de la réanimation, et ce, malgré un environnement plus restreint.
Thème 3 : Le niveau de confiance en la pratique professionnelle des infirmiers et médecins face à la présence de la famille.

Quatorze articles évaluent le niveau de la confiance en la pratique professionnelle. Twibell et al. (2018) définissent le concept de la confiance en la pratique professionnelle comme étant la capacité d'une personne à adopter un comportement. Le niveau de confiance en la pratique professionnelle est une dimension importante dans la pratique d'inclusion des familles lors d'une réanimation. En effet, Twibell et al. (2008) ont constaté que le niveau de confiance en la pratique professionnelle des infirmiers face à la présence familiale a un lien direct avec l'inclusion de la famille : plus le taux de confiance en la pratique professionnelle sera élevé, plus les médecins et infirmiers seront en mesure d'inviter la famille.

Plusieurs barrières peuvent affecter le niveau de confiance en la pratique professionnelle et donc limiter l'inclusion de la famille; la nouveauté de cette pratique et l'absence d'autorité discrétionnaire (Fulbrook et al., 2005). Holzhauser et Finucane (2007) montrent que lorsque l'opinion du personnel quant à la présence familiale est favorable, le degré d'aisance de ces derniers augmente. Il est alors envisagé qu'ils peuvent acquérir ainsi l'adoption de cette pratique inclusive dans leurs milieux. En se référant à la définition de Twibell et al. (2018), leur niveau de confiance en la pratique professionnelle serait ainsi renforcé. Selon Tomlinson et al. (2010) une plus grande sensibilisation à la population sur le processus de réanimation augmenterait une prise de décision favorable quant à la présence familiale chez les infirmiers. Ils seront ainsi davantage aptes à inclure la famille lors des manœuvres de réanimation, renforçant ainsi leur niveau de confiance en la pratique professionnelle.

Le niveau de confiance en la pratique professionnelle dépend de l'expérience du professionnel. Tudor et al. (2014) ont utilisé dans leur étude les échelles de Twibell et al. (2008). Ces derniers concluent que les infirmiers qui avaient déjà procédé à une invitation de la famille ou qui désiraient qu'un membre de leur propre famille insiste à leur réanimation éventuelle présentent un niveau de confiance en la pratique professionnelle plus élevé (Tudor et al., 2014). Howlett et al. (2010) rapportent que $79 \%$ des quatorze médecins expérimentés d'un centre régional de traumatologie présentaient une attitude plus favorable à l'égard de la présence familiale que $19 \%(\eta=22)$ des résidents moins expérimentés. Le niveau de confiance en la pratique professionnelle dépend aussi de l'expérience de l'inclusion des familles lors d'une précédente réanimation. Récemment, Twibell et al. (2018) montrent que les médecins ayant déjà invité des membres de la famille pendant la réanimation obtiennent une note significativement plus élevée sur l'échelle de la confiance en la pratique professionnelle que ceux qui n'avaient jamais invité des membres de la famille. Porter et al. (2014) montrent également que si le personnel a déjà inclus la famille il devient alors plus susceptible de le faire à nouveau. Ces mêmes auteurs soulèvent que l'enseignement pourrait conduire à davantage d'attitudes en faveur de la présence de la famille (Porter et al., 2014). Devant ces attitudes favorables, le personnel aura davantage tendance à inclure les familles lors des manœuvres de réanimation. Leur niveau de confiance en la pratique professionnelle sera ainsi renforcé. 
Twibell et ses collaborateurs (2018) ont mené des études à partir d'un outil mesurant le niveau de confiance en la pratique professionnelle chez les infirmiers et médecins. Ces auteurs rapportent que les infirmiers qui perçoivent plus d'avantages et moins d'inconvénients perçoivent une plus grande confiance en la pratique professionnelle. Les infirmiers exerçant à l'urgence ont davantage de confiance (Twibell et al., 2008) ainsi que celle qui possèdent un certificat de spécialité (Tudor et al., 2014).

\section{Discussion et recommandations}

Cette revue intégrative présente une recension des écrits de la perception des membres de l'équipe interprofessionnelle face à la présence de la famille lors des manœuvres de réanimation (voir tableau 1). Un constat s'impose : les études recensées portent uniquement sur les perceptions des infirmiers et des médecins. Or, ces professionnels de la santé ne sont pas seuls à œuvrer en salle de réanimation : ils travaillent en étroite collaboration avec une équipe interprofessionnelle constituée d'infirmiers, de médecins, d'inhalothérapeutes et de préposés aux bénéficiaires (Vincent, 2006). D'ailleurs, l'Ordre des infirmières et infirmiers du Québec (OIIQ) met en avant l'importance de cette collaboration interprofessionnelle par une prise de position où il affirme que cette dernière devrait exister dans tous les milieux de soins (OIIQ 2015). Cinq ans plus tard, l'OIIQ réitère sa position face à l'approche collaborative en continuant de souligner cette dimension importante en plaidant de nouveau en faveur de la collaboration interprofessionnelle (OIIQ 2020). Dans ce même ordre d'idée, l'Université de Montréal, adopte une vision plus large de cette approche collaborative. En effet, elle intègre le patient et sa famille comme partenaires de soins. Elle définit d'ailleurs le travail d'équipe comme étant la dynamique régnante entre le patient, sa famille et les intervenants (Direction collaboration et partenariat patient [DCPP] et Comité interfacultaire opérationnel de formation à la collaboration interprofessionnelle en partenariat avec le patient de l'Université de Montréal [CIO-UdeM, 2016]). Il est donc important de souligner que l'approche collaborative devrait occuper une place importante dans la pratique interprofessionnelle lors de la réanimation. Cette approche collaborative permet d'appuyer les résultats de cette revue intégrative en soulignant l'importance d'inclure le patient et sa famille comme partenaires de soins. Ainsi, cette approche collaborative pourrait servir de point d'ancrage aux gestionnaires pour créer des politiques institutionnelles encadrant la pratique d'inclusion des familles lors des manœuvres de réanimation à l'urgence.

Les résultats de la revue intégrative montrent également que les perceptions favorables au regard de l'inclusion des familles suscitent l'invitation des familles par l'équipe interprofessionnelle. Il a aussi été souligné que ces perceptions permettraient une plus grande confiance en la pratique professionnelle des professionnels au regard de l'inclusion des familles. Ainsi, informer les étudiants lors de la formation initiale sur l'ensemble des bénéfices que soulignent cette revue intégrative des écrits susciterait certainement des avis favorables quant à cette pratique. Leur niveau de confiance en soi pourrait ainsi être influencé positivement. Néanmoins, apporter uniquement de l'information aux étudiants quant aux bénéfices de l'inclusion des familles ne serait sans doute pas suffisant pour assurer une mise en pratique de cette inclusion dans les milieux de soins. Toutefois, il a aussi été vu que l'expérience professionnelle influence la perception des bénéfices pour l'équipe interprofessionnelle en présence des familles lors des manœuvres de réanimation. De ce fait, il serait pertinent de donner la possibilité aux étudiants de se pratiquer aux manœuvres de réanimation en présence d'un proche du patient, lors des séances dans les centres de simulation intégrés. En effet, il a été démontré qu’après une formation basée sur la simulation, une meilleure application des recommandations lors des manœuvres de réanimation est observée (Boet et al., 2013). De plus, la simulation permettrait de réduire l'anxiété des étudiantes (Roh, 2014). Cela est d'autant plus intéressant face aux résultats de la revue intégrative qui montrent que l'inclusion des familles pouvait être source de stress et d'anxiété chez l'équipe interprofessionnelle.

Les retombées concernent également la formation en cours d'emploi. Devant ce même constat, il serait intéressant de prévoir des séances dans un centre de simulation pour les professionnels de la santé. En effet, l'OIIQ souligne que la formation continue permet la mise à jour des connaissances, le maintien des compétences mais également l'évolution et le développement des pratiques de soins (OIIQ 2011). De plus, il a été montré que l'expérience professionnelle peut s'acquérir par la formation et par l'apprentissage en situation de travail, soit la simulation (Grasser et Rose, 2000). Ainsi, comme le montre les résultats de la revue intégrative, l'expérience acquise pourrait renforcer le niveau de confiance en la pratique professionnelle de l'équipe interprofessionnelle suscitant ainsi des avis favorables quant à l'inclusion des familles.

Il est également possible de constater à la lumière de cette revue intégrative des écrits quaucune recherche ne semble porter sur le lien entre les perceptions des professionnels de la santé et l'organisation du travail. Pourtant, dans une optique de gestion hospitalière, tout porte à croire que ces recherches permettraient possiblement d'instaurer une culture d'inclusion des familles et d'instaurer une culture collaborative. Afin d'appuyer cette hypothèse, il est important de souligner que l'Organisation Mondiale de la Santé (OMS) présente plusieurs avantages de cette culture collaborative comme une réponse davantage complète et mieux intégrée aux besoins du patient et de ses proches mais aussi une meilleure gestion des épisodes de soins par les intervenants (OMS, 2010).

\section{Limites}

Cet article comporte différentes limites. Premièrement, cette revue intégrative n'a pas retenu d'article ciblant le point de vue des gestionnaires ou de décideurs politiques. La perception de ces derniers n'était pas incluse dans les questions de recherche. Il aurait pu être pertinent d'en tenir compte face à l'importance de prôner une culture d'inclusion de la famille dans les milieux de soins. Cette revue intégrative n'a également pas retenu d'article ciblant le point de vue de l'équipe interprofessionnelle œuvrant dans les urgences pédiatriques en se limitant aux adultes. De plus, la méthode d'analyse des données basée sur les thèmes de Twibell et al. (2008) entraîne un biais dans l'émergence d'autres thèmes. Une dernière limite serait de ne pas avoir différencié les 
perceptions de l'équipe interprofessionnelle selon leur milieu d'exercice. Ainsi, cette revue intégrative ne permet pas de soulever des différences potentielles de perceptions selon le milieu d'exercice.

\section{Conclusion}

Les résultats de cette revue intégrative offrent des nouvelles pistes d'interventions pour améliorer la pratique d'inclusion des familles lors des manœuvres de réanimation. Une attention particulière devrait être portée sur la formation initiale chez les futurs professionnels de la santé mais également sur la formation continue. En effet, faire valoir les bénéfices de cette inclusion auprès des étudiants et auprès de l'équipe interprofessionnelle permettrait de susciter davantage d'avis favorables quant à cette pratique. En parallèle, instaurer l'inclusion de la famille dans les centres de simulation intégrés développera l'expérience des soignants quant à cette pratique de soins tout en renforçant leur confiance en la pratique professionnelle. Enfin, cet article suscite un début de réflexion chez les gestionnaires et décideurs sur l'importance d'instaurer une culture collaborative ainsi qu'une approche centrée sur le patient dans les milieux de soins.

\section{Implications pour les infirmiers}

Les retombées de cette revue intégrative des écrits peuvent se décliner au niveau de la pratique clinique, de la formation, de la recherche, de la politique et de la gestion.

1. Au niveau de la gestion, la retombée la plus importante se situe au niveau du leadership infirmier dans la promotion de l'inclusion des familles lors des manœuvres de réanimation. À la lumière des résultats, il s'avère que l'élaboration de protocoles et politiques de gestion permettrait de favoriser cette pratique clinique d'inclusion des familles. Devant ces perceptions divergentes, il devient souhaitable que le gestionnaire facilite des rencontres interprofessionnelles lors de l'élaboration de protocoles afin d'ouvrir le dialogue sur les perceptions entre les membres de l'équipe.

2. Une sensibilisation constante de l'équipe interprofessionnelle quant à l'inclusion des familles lors de manœuvres permettrait de contribuer au développement professionnel de l'équipe.

3. Finalement, sur le plan de la recherche, des études sont nécessaires pour documenter la perception de chaque membre de l'équipe interprofessionnelle afin que la voix des préposés aux bénéficiaires et des inhalothérapeutes soient également entendues. Ceci permettrait de mieux comprendre les éléments favorisant l'inclusion des familles.

\section{Les auteurs}

Quentin Bevillard-Charrière, inf. M. Sc., Faculté des Sciences Infirmières, Université de Montréal

quentin.bevillard-charriere@umontreal.ca

Steve Gagné, inf., MScN, CNCC (C), Conseiller en soins spécialisés - Développement de la pratique et des compétences, ChercheurProfessionnel de la santé au Centre de recherche du CHUM, Centre Hospitalier de l'Université de Montréal, Infirmier clinicien spécialisé associé, Faculté des sciences infirmières, Université de Montréal

steve.gagne.chum@ssss.gouv.qc.ca

Laurence Bernard, inf. Ph.D, Professeure agrégée, Faculté des Sciences Infirmières, Université de Montréal

laurence.bernard@umontreal.ca

\section{REFERENCES}

Asencio-Gutiérrez, J. M. et Reguera-Burgos, I. (2017). The opinion of health professionals regarding the presence of relatives during cardiopulmonary resucitation. Enfermería Intensiva (English Ed.), 28(4), 144-159. https://doi.org/10.1016/j.enfie.2017.10.006

Axelsson, Å. B., Fridlund, B., Moons, P., Mårtensson, J., op Reimer, W. S., Smith, K., Strömberg, A., Thompson, D., et Norekvål, T. M. (2010). European cardiovascular nurses' experiences of and attitudes towards having family members present in the resuscitation room. European Journal of Cardiovascular Nursing, 9(1), 15-23. https://doi.org/10.1016/j.ejcnurse.2009.10.001

Boet, S., Granry, J. C., et Savoldelli, G. (Eds.). (2013). La simulation en santé de la théorie à la pratique. Paris: Springer.

Chapman, R., Bushby, A., Watkins, R. et Combs, S. (2014). Australian emergency department health professionals' reasons to invite or not invite family witnessed resuscitation: A qualitative perspective. International Emergency Nursing, 22(1), 18-24. https://doi. org/10.1016/j.ienj.2013.03.008

DCPP et CIO-UdeM. (2016). Référentiel de compétences de la Pratique collaborative et $d u$ Partenariat patient en santé et services sociaux. Montréal, Québec : Université de Montréal.

Emergency Nurses Association. (2012). Clinical practice guideline: Family presence during invasive procedures and resuscitation. https://www.ena.org/practice-research/CPG/Documents/ FamilyPresenceCPG.pdf

Fell, O. P. (2009). Family presence during resuscitation efforts. Nursing Forum, 44(2), 144-150. https://doi. org/10.1111/j.1744-6198.2009.00136.x
Fisher, C., Lindhorst, H., Matthews, T., Munroe, D. J., Paulin, D. et Scott, D. (2008). Nursing staff attitudes and behaviours regarding family presence in the hospital setting. Journal of Advanced Nursing, 64(6), 615-624.

Fulbrook, P., Albarran, J. W. et Latour, J. M. (2005). A European survey of critical care nurses' attitudes and experiences of having family members present during cardiopulmonary resuscitation. International Journal of Nursing Studies, 42(5), 557-568. https:// doi.org/10.1016/j.ijnurstu.2004.09.012

García-Martínez, A. L. et Meseguer-Liza, C. (2018). Emergency nurses' attitudes towards the concept of witnessed resuscitation. Revista Latino-Americana de Enfermagem, 26(0). https://doi. org/10.1590/1518-8345.1382.3055

Gomes, B. D., Dowd, O. P. et Sethares, K. A. (2019). Attitudes of community hospital critical care nurses toward family-witnessed resuscitation. American Journal of Critical Care, 28(2), 142-148. https://doi.org/10.4037/ajcc2019162

Grasser, B., et Rose, J. (2000). L'expérience professionnelle, son acquisition et ses liens à la formation. Formation emploi, 71(1), 5-19.

Holzhauser, K. et Finucane, J. (2007). Staff attitudes to family presence during resuscitation. Australasian Emergency Nursing Journal, 10(3), 124-133. https://doi.org/10.1016/j.aenj.2007.06.001

Holzhauser, K. et Finucane, J. (2008). Part B: A survey of staff attitudes immediately post-resuscitation to family presence during resuscitation. Australasian Emergency Nursing Journal, 11(3), 114122. https://doi.org/10.1016/j.aenj.2008.05.002 
Howlett, M. S. L., Alexander, G. A. et Tsuchiya, B. (2010). Health care providers' attitudes regarding family presence during resuscitation of adults: An integrated review of the literature. Clinical Nurse Specialist, 24(3), 161-174. https://doi.org/10.1097/ NUR.0b013e3181dc548a

Jabre, P., Belpomme, V., Azoulay, E., Jacob, L., Bertrand, L., Lapostolle, F., Tazarourte, K., Bouilleau,G., Pinaud, F., Broche, C., Normand, D., Baubet, T., Ricard-Hibon, A., Istria, J., Beltramini, A., Alheritiere, A., Assez, N., Nace, L., Vivien, B., ... Adnet, F.. (2013). Family presence during cardiopulmonary resuscitation. New England Journal of Medicine, 368(11), 1008-1018.

Jensen, L. et Kosowan, S. (2011). Family presence during cardiopulmonary resuscitation: Cardiac health care professionals' perspectives. Canadian Journal of Cardiovascular Nursing, 21(3).

Knott, A. et Kee, C. C. (2005). Nurses' beliefs about family presence during resuscitation. Applied Nursing Research, 18(4), 192-198. https://doi.org/10.1016/j.apnr.2005.07.002

Köberich, S., Kaltwasser, A., Rothaug, O. et Albarran, J. (2010). Family witnessed resuscitation - experience and attitudes of German intensive care nurses. Nursing in Critical Care, 15(5), 241-250. https://doi.org/10.1111/j.1478-5153.2010.00405.x

Loiselle, C. G. et Profetto-McGrath, J. (2007). Méthodes de recherche en sciences infirmières. Approches quantitatives et qualitatives. Adaptation française de Polit et Beck. Montréal, Québec : ERPI

MacLean, S. L., Guzzetta, C. E., White, C., Fontaine, D., Eichhorn, D. J., Meyers, T. A. et Désy, P. (2003). Family presence during cardiopulmonary resuscitation and invasive procedures: Practices of critical care and emergency nurses. Journal of Emergency Nursing, 29(3), 208-221.

Mason, D. J. (2003). Family presence: Evidence versus tradition. American Journal of Critical Care, 12(3), 190-192.

McClement, S. E., Fallis, W. M. et Pereira, A. (2009). Family presence during resuscitation: Canadian critical care nurses' perspectives. Journal of Nursing Scholarship, 41(3), 233-240. https://doi. org/10.1111/j.1547-5069.2009.01288.x
Meyers, T. A., Eichhorn, D. J., Guzzetta, C. E., Clark, A. P., Klein, J. D., Taliaferro, E. et Calvin, A. (2000). Family presence during invasive procedures and resuscitation: The experience of family members, nurses, and physicians. AJN The American Journal of Nursing, 100(2), 32-43.

Mian, P., Warchal, S., Whitney, S., Fitzmaurice, J. et Tancredi, D. (2007). Impact of a multifaceted intervention on nurses' and physicians' attitudes and behaviors toward family presence during resuscitation. Critical Care Nurse, 27(1), 52-61.

Miller, J. H. et Stiles, A. (2009). Family presence during resuscitation and invasive procedures: The nurse experience. Qualitative Health Research, 19(10), 1431-1442. https://doi. org/10.1177/1049732309348365

Organisation Mondiale de la Santé. (2010). Framework for action on interprofessional education and collaborative practice (No. WHO/ $\mathrm{HRH} / \mathrm{HPN} / 10.3)$. Organisation Mondiale de la Santé.

Ordre des Infirmières et Infirmiers du Québec. (2011). Vers une culture de formation continue pour la profession infirmière au Québec: Document d'orientation.

Ordre des Infirmières et Infirmiers du Québec. (2015). Énoncé de position conjoint sur la collaboration interprofessionnelle : Rehausser la qualité et la sécurité des soins (OIIQ-CMQ-OPQ). https://www. oiiq.org/enonce-de-position-conjoint-sur-la-collaborationinterprofessionnelle-rehausser-la-qualite-et-la-securite-des-soinsoiiq-cmq-opq-

Ordre des Infirmières et Infirmiers du Québec. (2020, 19 mai). Réplique: un plaidoyer en faveur de la collaboration interprofessionnelle. La Presse, https://www.lapresse.ca/debats/ opinions/202005/18/01-5274067-replique-un-plaidoyer-enfaveur-de-la-collaboration-interprofessionnelle.php

Look for supplemental materials such as author interviews and podcasts at www.CJEN.ca

The Canadian Journal of Emergency Nursing (CJEN) is the Official Journal of the National Emergency Nurses Association (NENA) of Canada. This article has been made available at no cost in partnership with NENA and the University of Alberta Libraries. 